\title{
Validação Interna de Base de Conhecimentos utilizando Modelagem Matemática Bayesiana $x$ Conexionista
}

\author{
Lucimar Maria Fossatti de Carvalho ${ }^{1}$ \\ Augusto Gai Fuão ${ }^{1}$ \\ Hugo José Teixeira de Carvalho ${ }^{2}$
}

\begin{abstract}
Resumo: Neste artigo, investigamos o aprendizado de máquina baseado na modelagem matemática envolvendo modelos bayesianos $x$ conexionistas, aplicado no auxílio ao diagnóstico de eventos epilépticos (EEs) e eventos não epilépticos (ENEs). Para esta finalidade alguns algoritmos de aprendizado de máquina foram comparados incluindo o aprendizado conexionista através das regras Delta Generalizada, Hebb, Oja, juntamente com o aprendizado em redes bayesianas. Foram considerados para este estudo 122 pacientes os quais apresentavam sintomas relacionados com EEs e ENEs. Os resultados empíricos desta pesquisa indicam que: (1) ambas as abordagens bayesianas e conexionistas são bastante similares, mostrando que os resultados são coerentes, mesmo utilizando heurísticas distintas; (2) o índice de acertos de casos positivos (EEs), por meio da modelagem bayesiana pode estar relacionado com a facilidade de implementação das probabilidades condicionais. Concluiu-se, portanto, que os modelos investigados fornecem informações importantes para o desenvolvimento de novas pesquisas tanto na área bayesiana como na área conexionista.
\end{abstract}

Palavras-chave: Aprendizagem de máquina. Redes bayesianas. Redes neurais artificiais.

\begin{abstract}
In this article, we investigated the machine learning based on the mathematics modeling bayesian and connectionist, applied to support in the diagnostic of epileptic events (EEs) and non-epileptic events (NEEs). To this end, some algorithms of machine learning were compared including the connectionist learning through Generalized Delta, Hebb and Oja rules learning, with the learning in Bayesian Networks. There were considered to this study 122 patients which showed symptoms related to EEs and NEEs. The empiric results of this search indicate that: (1) both bayesian and connectionist approaches are too similar, revealing consistent results, even using distinct heuristics; (2) the indices of positive cases (EEs), from the bayesian modeling may be related to the facility of the implementation of the conditional probabilities. It was concluded, therefore that the investigated models give important information to the development of new searches as in the bayesian field as in the connectionist field.
\end{abstract}

Keywords: Learning machine. Bayesian networks. Artificial neural networks.

\section{Introdução}

Epilepsia é uma condição do sistema nervoso central que afeta em torno de cinquenta milhões de pessoas no mundo e em qualquer tempo. Consiste duma desordem neurológica crônica caracterizada por crises recorrentes e não provocadas [1]. Crises epilépticas são eventos clínicos que refletem uma disfunção temporária de um conjunto de neurônios de parte do encéfalo, conhecidas como "crises focais", ou de uma área mais extensa, envolvendo simultaneamente os dois hemisférios cerebrais, chamadas de "crises generalizadas" [2]. O desafio diagnóstico são os chamados "pacientes mistos", portadores de eventos epilépticos (EEs) e de eventos não epilépticos (ENEs) [3].

\footnotetext{
${ }^{1}$ Curso de Ciência da Computação, UPF, Campus 1 - BR 285 - Passo Fundo (RS) - Brasil 
A área da inteligência artificial (IA) possui métodos e técnicas para trabalhar questões complexas, principalmente no auxílio ao diagnóstico médico. Dessa forma, surge a necessidade de se investigar a aplicabilidade de diferentes modelagens matemáticas relacionadas com a área da inteligência computacional (IC), as quais podem ajudar a classe médica na solução de problemas complexos, levando a um índice maior de acertos. A área da IC compreende a teoria e a aplicação de técnicas computacionais inspiradas em fenômenos naturais que incluem redes neurais artificiais (RNAs), lógica Fuzzy e computação evolucionária [4]. Características básicas desses sistemas encontram-se na representação do conhecimento inexato, que seria impossível de representar por meio das técnicas tradicionais.

Atualmente existe um grande interesse na aplicação de técnicas de aprendizagem de máquina aplicadas no auxílio ao diagnóstico médico. Entre as técnicas não lineares pesquisadas encontra-se a área de RNAs, com os trabalhos de [5], [6], [7], [8], [9] e [10], incluindo a área de redes bayesianas (RB) ([11], [12], [13], [14] e [15]), entre outros.

Nesta pesquisa são investigadas a aprendizagem de máquina baseada na modelagem matemática bayesiana $x$ conexionista.

\section{Modelagem Matemática Bayesiana}

Segundo Rich e Knight [16], RBs são grafos acíclicos dirigidos que representam dependências entre variáveis num modelo probabilístico. Os nós representam as variáveis aleatórias com medidas de incerteza associadas, ao passo que os arcos representam a influência causal direta entre as variáveis conectadas, e a força dessas influências é quantificada por probabilidades condicionais.

As RBs são adequadas para trabalhar com dados sob condições de incerteza para representar conhecimento probabilístico e seu aprendizado está diretamente relacionado com as probabilidades condicionais e incondicionais que ocorrem entre as variáveis de entrada [12]. Por ser fundamentado na teoria da probabilidade, o raciocínio probabilístico é uma das grandes vantagens das RBs, ou seja, é possível tomar decisões racionais mesmo quando não há informações suficientes para provar que uma determinada ação funcionará corretamente.

Segundo Dutra e Marques [15], as pesquisas sobre RBs tiveram início na década de 1990. A partir daí, as RBs estão sendo utilizadas para solucionar vários tipos de problemas em diversas áreas. Também conhecidas como "redes causais", as RBs são caracterizadas por um poderoso formalismo que representa o conhecimento em um determinado domínio e também pelas incertezas associadas a este domínio.

Para construir uma RB para auxílio ao diagnóstico médico, é necessária a realização das seguintes etapas: (1) obtenção de dados, (2) modelagem do domínio, (3) refinamento da rede inicial, e (4) validação da rede com relação a novos casos [17]. Segundo a autora, dois tipos de cálculos são realizados por uma RB: (1) atualização de crenças, que consiste no cálculo de probabilidade das variáveis aleatórias e (2) revisão de crenças, a qual se refere à obtenção das probabilidades das hipóteses, juntamente com a identificação da hipótese diagnóstica que apresenta maior valor. Em uma RB pode-se observar a propagação de uma informação de entrada em toda a rede, permitindo, dessa forma, observar a quantidade de informação que trafega na rede. Este processo é realizado por meio do teorema de Bayes.

\subsection{Teorema de Bayes}

O teorema de Bayes constitui o mecanismo no qual a medida de certeza de uma determinada hipótese $H$ (diagnóstico) seja calculado, dado uma evidência $E$ (sintoma), a qual condicionalmente deverá ser dependente de $H$ [18].

As probabilidades bayesianas são consideradas uma teoria estatística de evidências. A noção fundamental da estatística bayesiana é fundamentada na probabilidade condicional, como pode ser observado em (1):

$$
P(H / E)
$$

Segundo Rich e Knight [16], esta expressão está relacionada com a probabilidade da hipótese $H$, dado que observamos a evidência $E$. Para computá-la, é preciso levar em consideração a probabilidade prévia de $H$ e até que ponto $E$ fornece evidências de $H$. 
Onde:

$P\left(H_{i} \mid E\right)=$ probabilidade da hipótese $H_{i}$ ser verdadeira, dada a evidência $E$;

$P\left(E \backslash H_{i}\right)=$ probabilidade de observar a evidência $E$, dado que a hipótese $H_{i}$ seja verdadeira;

$P\left(H_{i}\right)=$ probabilidade a priori de que a hipótese $i$ seja verdadeira na ausência de qualquer evidência específica. Esta probabilidade é chamada de "probabilidade prévia".

O teorema de Bayes baseado em [18] pode ser observado em (2).

$$
P(H / E)=\frac{P(E / H) * P(H)}{P(E)}
$$

De acordo com a fórmula, a probabilidade de uma hipótese $H$ ocorrer, dado uma evidência $E$, é definida como a probabilidade das evidências dadas as hipóteses multiplicada pelas probabilidades das hipóteses. Este valor é dividido pela probabilidade das evidências.

De acordo com Dutra e Marques [15], para aplicação da regra de Bayes são necessários três termos: uma probabilidade condicional e duas incondicionais.

Considera-se o seguinte exemplo de diagnóstico médico: um médico especialista sabe que um paciente com uma crise generalizada após perder a consciência (causado por um desmaio) pode ter "mordido a língua" em $50 \%$ dos casos. Porém, o médico também conhece algumas probabilidades incondicionais, pelas quais uma crise generalizada atinge 1/50000 de indivíduos, e a probabilidade, a priori, de alguém ter "mordido a língua" durante o desmaio é de $1 / 20$."

Consideram-se então, $E($ evidência $=$ morder a língua $=$ sintoma $)$ e $H($ diagnóstico $=$ crise generalizada $)$, respectivamente, como a probabilidade incondicional de um paciente ter "mordido a língua" e a probabilidade incondicional de um paciente ter uma crise generalizada. Desta forma:

$$
\begin{aligned}
& P(E / H)=1 / 2 \text { (probabilidade de ter "mordido a língua" tendo uma crise generalizada) } \\
& P(H)=1 / 50000=0,00002 \\
& P(E)=1 / 20=0,05
\end{aligned}
$$

Aplicando a regra de Bayes, como pode ser observado em (3):

$$
\begin{gathered}
P(H / E)=(P(E / H) * P(H)) / P(E) \\
P(H / E)=((1 / 2) *(1 / 50000)) /(1 / 20)=0,00001 / 0,05=0,0002=1 / 5000
\end{gathered}
$$

Este exemplo mostra que é esperado que apenas um em 5.000 pacientes que tenham "mordido a língua" seja uma crise generalizada. Observe-se que, apesar de o paciente ter "mordido a língua" nos casos de crise generalizada $(0,5)$, a probabilidade de ele ter uma crise generalizada continua pequena. Isso ocorre pelo fato de a probabilidade incondicional do indivíduo ter "mordido a língua" ser maior que a probabilidade de ser uma crise generalizada.

\subsection{Construindo uma Rede Bayesiana}

Nesta pesquisa, para efeitos de comparação com os modelos conexionistas, foram coletadas informações a partir do médico especialista na área da neurologia ${ }^{3}$. A base de conhecimentos foi construída com a shell Netica [19], possuindo 122 pacientes cadastrados. As probabilidades de ocorrência dos sintomas foram definidas em conjunto com o médico neurologista. Foram analisadas as evidências (sintomas) de entrada para obter a definição das hipóteses de saída (diagnósticos).

Dessa forma, as características referentes aos ENEs implementadas nos modelos bayesianos foram: (1) Quadros clássicos de conversão, (2) Distúrbios de sensibilidade e da atividade sensorial, (3) Fatores emocionais/físicos, (4) Transtornos, (5) Síncopes, (6) Distúrbios. Já as características referentes aos EEs são: (7)

\footnotetext{
${ }^{3}$ Hugo José Teixeira de Caralho, médico neurologista do Hospital da Cidade e Hospital São Vicente de Paulo da cidade de Passo Fundo/RS.
} 
Sinais e sintomas parciais, (8) Durante o desmaio "viu" alguma coisa, (9) Durante o desmaio "ouviu" alguma coisa, (10) Durante o desmaio mordeu a língua, (11) Durante o desmaio machucou-se, (13) Tempo de duração do desmaio, (13) Eventos pós-críticos, e (14) Sinais e sintomas generalizados. Essas características estão relacionadas com as variáveis de entrada.

A variável de saída está relacionada com a definição do diagnóstico pelo especialista, ou seja, se o paciente apresentou um EE ou um ENE, de acordo com os sintomas apresentados.

Foram implementados três modelos de redes bayesianas: (1) considera apenas a presença ou ausência do sintoma (Sim/Não); (2) foi elaborado a partir da base de dados; (3) foi elaborado a partir da definição das variáveis pelo médico neurologista. Os modelos dois e três consideram para a implementação a frequência de ocorrência dos sintomas (às vezes, frequentemente e sempre). Os modelos bayesianos possuem 14 variáveis de entrada e uma variável de saída, definidas em conjunto com o médico especialista.

A Figura 1 mostra o primeiro modelo bayesiano implementado a partir da base de dados.

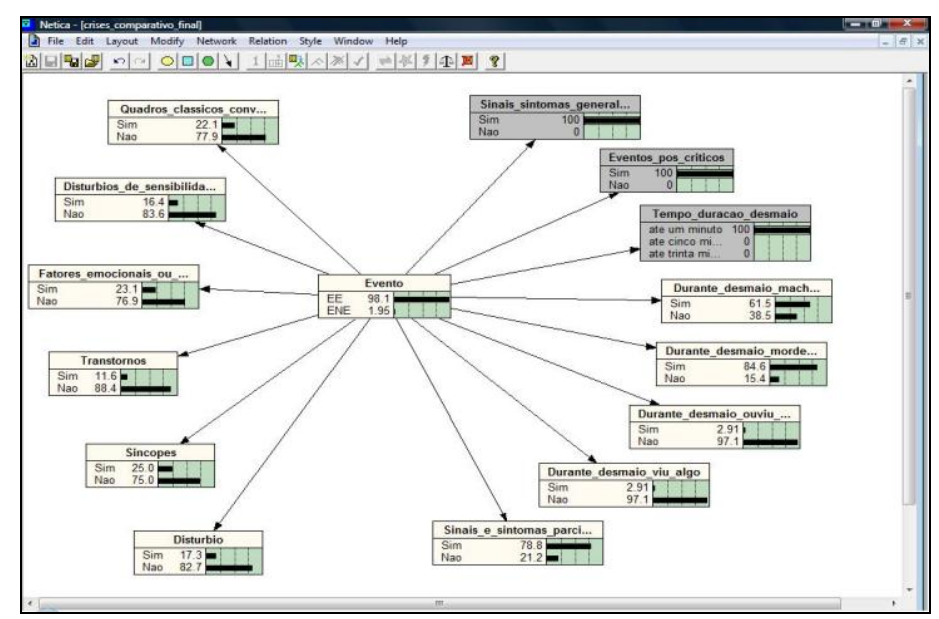

Figura 1. Primeiro Modelo Bayesiano

A Figura 1 mostra a saída da rede, de acordo com os sintomas apresentados pelo paciente, obtidos a partir da base de dados. Após os cálculos, observa-se que o paciente que apresenta os três sintomas (tempo de duração do desmaio até um minuto; eventos pós críticos e sinais e sintomas generalizados) possui 98,1\% de chances de ser um paciente com um EE.

A Figura 2 mostra o segundo modelo bayesiano implementado a partir da base de dados, incluindo as variáveis às_vezes, frequentemente e sempre.

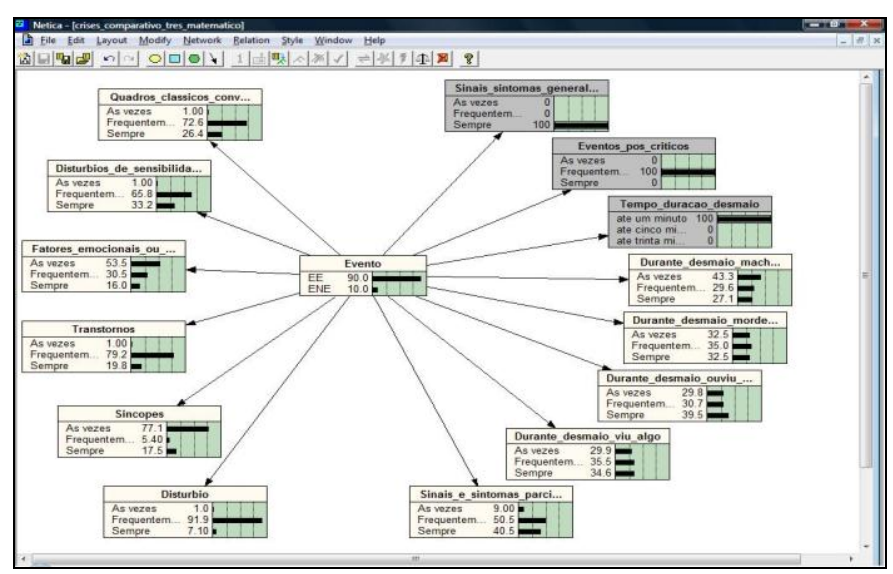

Figura 2. Segundo Modelo Bayesiano

De acordo com a Figura 2, observa-se que o paciente que apresenta os mesmos três sintomas do primeiro modelo possui $90,0 \%$ de chances de ter um EE. 
Perceberam-se neste modelo alguns problemas no funcionamento da rede, ou seja, como a base de dados dos pacientes é pequena, alguns sintomas não foram contemplados, recebendo o valor de $1 \%$, o que levou a rede a apresentar algumas incoerências. Mesmo com esses problemas, a rede obteve uma sensibilidade satisfatória, como é mostrado na seção dos resultados finais.

O terceiro modelo é semelhante ao segundo. Para a implementação desse modelo, utilizou-se a mesma base de testes do segundo modelo; a descrição das variáveis de entrada e da variável de saída também é a mesma do segundo modelo. A única alteração em relação ao segundo modelo é que as probabilidades condicionais foram obtidas a partir do especialista de domínio, como mostra a Figura 3.

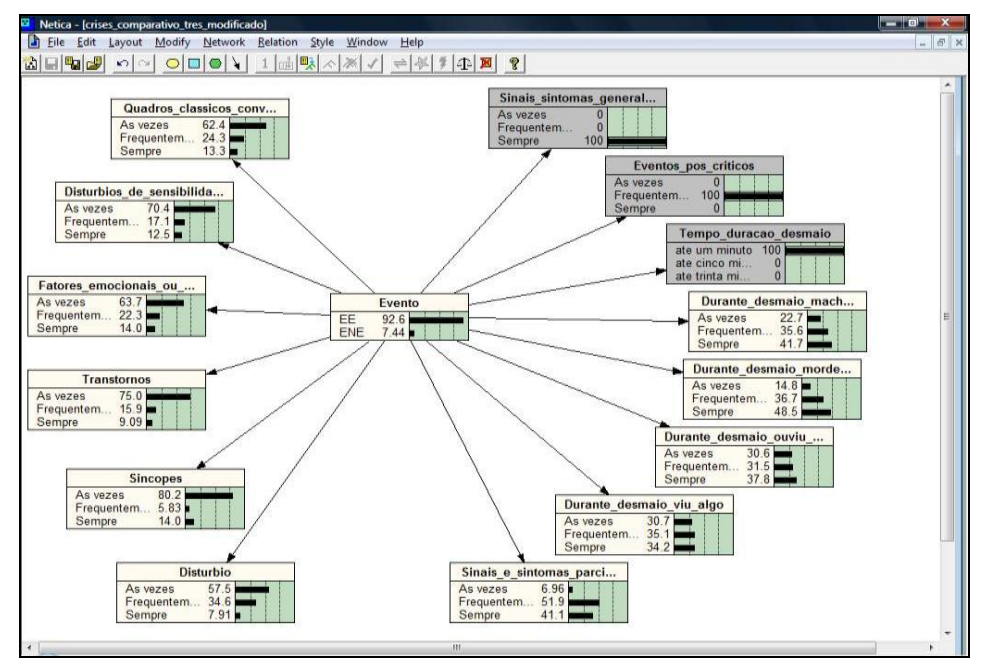

Figura 3. Terceiro Modelo Bayesiano

A Figura 3 mostra a saída da rede de acordo com os sintomas apresentados pelo paciente. Após os cálculos das probabilidades condicionais, observa-se que o paciente que apresentou os três sintomas possui $92,6 \%$ de chances de ser um paciente com EE. De acordo com a figura, o que diferiria o segundo modelo do terceiro é o fato de que no segundo, os valores de entrada de cada sintoma foram obtidos a partir da base de dados, ao passo que no terceiro foram obtidas com o auxílio do especialista.

Para efeitos de comparação, na implementação dos três modelos bayeseianos foi utilizada a mesma base de dados implementada nos trabalhos de [24], [25] e [28].

\section{Modelagem Matemática Conexionista}

As RNAs são modelos matemáticos que se assemelham às estruturas neurais biológicas, possuindo capacidade computacional adquirida por meio do aprendizado e generalização a partir de exemplos. Depois de treinada, o conhecimento da rede fica armazenado em um conjunto padrão de pesos, os quais são distribuídos por toda a rede, por meio das conexões entre seus neurônios [12].

De acordo com [20], as RNAs, estruturalmente conhecidas como modelos conexionistas, são sistemas paralelos e distribuídos compostos por unidades de processamento simples que calculam determinadas funções matemáticas. As unidades são dispostas em uma ou mais camadas e interligadas por muitas conexões, geralmente unidimensionais. A maioria dos modelos utiliza essas conexões associadas a pesos, os quais possuem a função de armazenar conhecimento.

A utilização de RNAs tem como objetivo solucionar problemas de reconhecimento de padrões que geralmente são baseados em um conjunto de informações previamente conhecidas. Possuem a capacidade de adquirir conhecimento através da experiência e da observação e, com base nisso, realizar classificações e generalizações de classes de dados [21].

Segundo os autores [20], as RNAs tentam reproduzir as funções das redes biológicas, porém, do ponto de vista fisiológico, ainda diferem das redes biológicas. Mesmo assim, possuem algumas características comuns: os dois sistemas são baseados em unidades de computação paralela e distribuída que se comunicam por meio de 
conexões sinápticas; possuem detectores de características, redundância e modularização das conexões. Portanto, acredita-se que estruturas encontradas nos sistemas biológicos podem inspirar o desenvolvimento de novas arquiteturas para modelos de RNAs no futuro.

Nesta pesquisa, as comparações foram realizadas com RNAs implementadas com o Multilayer Perceptron (MLP), a partir do algoritmo de aprendizado backpropagation. As redes MLP são compostas de neurônios com funções de ativação nas camadas intermediárias e de saída. São redes constituídas de uma camada de entrada, que recebe estímulos do meio externo, uma ou mais camadas intermediárias (ocultas) e uma camada de saída. O sinal de entrada se propaga para frente através da rede, camada por camada [22].

É importante observar que, em alguns casos, a utilização de duas ou mais camadas intermediárias poderá facilitar o treinamento da rede. Quanto maior for o número de neurônios, maior será a complexidade da rede e maior a sua abrangência em termos de soluções possíveis. Em razão disso, a determinação do número de neurônios é fundamental na modelagem [20].

A Figura 4 apresenta uma rede MLP, a qual utiliza o algoritmo de aprendizado backpropagation.

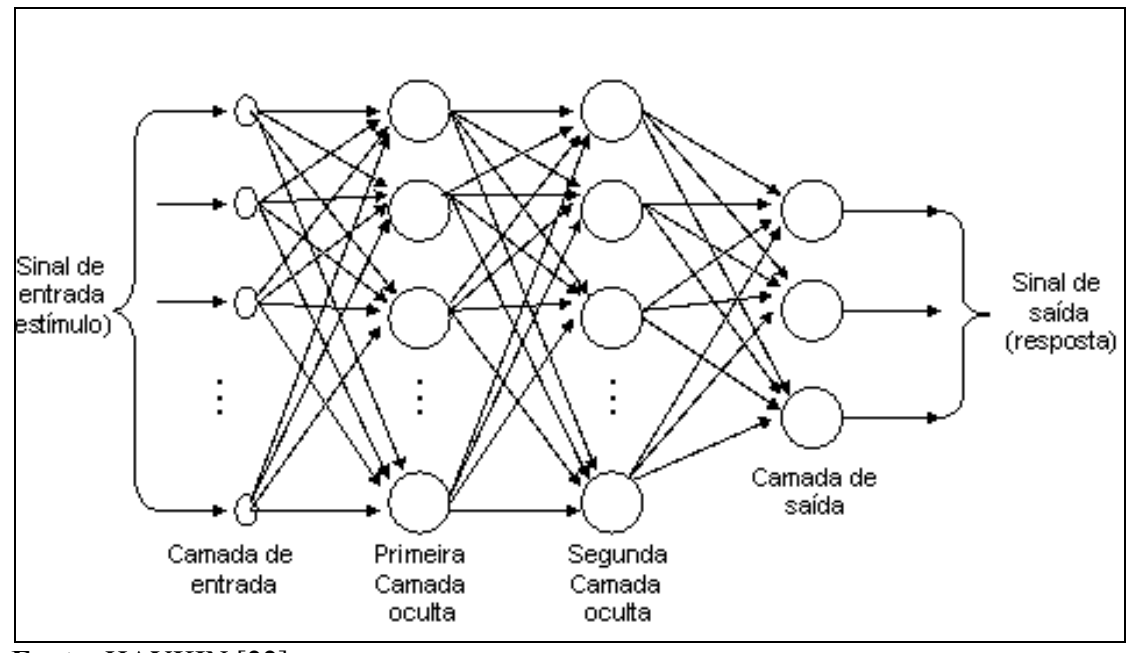

Fonte: HAYKIN [22]

Figura 4. Perceptron de Múltiplas Camadas

De acordo com a Figura 4, o MLP contém quatro camadas: uma de entrada, duas camadas intermediárias e uma camada de saída. A camada de entrada recebe os estímulos externos e a camada de saída fornece a saída da rede.

Uma rede MLP possui três características [22]:

1) o modelo de cada neurônio da rede inclui uma função de ativação não linear;

2) a rede possui uma ou mais camadas de neurônios ocultos, com capacidade de aprender tarefas complexas, extraindo progressivamente as características mais significativas dos padrões de entrada;

3) a rede possui um alto grau de conectividade, de modo que qualquer alteração na conectividade da rede requer mudanças nas conexões sinápticas ou nos pesos.

O algoritmo de aprendizado backpropagation baseia-se no aprendizado supervisionado por correção de erros, proposto por [23]. É constituído de duas fases distintas: propagação ou avanço, conhecida como feedforward, e retropropagação ou retrocesso, conhecida como backforward. Na fase de propagação, um padrão de atividade é aplicado aos nós sensoriais da rede e seu efeito se propaga através da rede, camada por camada, até que um conjunto de saídas seja produzido como a resposta final da rede. Nessa fase, os pesos sinápticos da rede são fixos. Já, na fase de retropropagação, os pesos sinápticos são ajustados de acordo com uma regra de correção de erro; a saída obtida é subtraída da saída desejada para produzir um sinal de erro. Esse sinal de erro é propagado para trás através da rede, influenciando na correção dos pesos na direção contrária às conexões sinápticas. Os pesos sinápticos são ajustados para que a resposta real da rede fique cada vez mais próxima da resposta desejada [22]. 
Nesta pesquisa os modelos conexionistas comparados possuem as mesmas características dos modelos bayesianos, ou seja, 14 neurônios de entrada, representando os sintomas apresentados pelos pacientes, e uma variável de saída, a qual determina a ocorrência de um EE ou um ENE. Tanto a base de treinamento como a base de teste possuem 122 registros.

\section{Resultados}

$\mathrm{Na}$ modelagem matemática das RBs foram implementados três modelos bayesianos, os quais são comparados com os resultados de três abordagens conexionistas, ambas utilizando uma rede MLP através do algoritmo de aprendizado backpropagation: (1) implementação de uma RNA utilizando as regras de aprendizado: (a) Delta Generalizada; (b) Hebb e (c) Oja [24]; (2) um sistema neuro-difuso implementado por [25] a partir dos neurônios difusos propostos por [26] e [27] e (3) proposto por [28], que implementou um sistema seuro-difuso com as operações aritméticas fuzzy $\mathrm{min} / \mathrm{max}$, utilizando a arquitetura NEFCLASS [29].

$\mathrm{Na}$ sequência, descrevem-se os resultados obtidos com a validação interna da base de conhecimentos utilizando os três modelos bayesianos.

\subsection{Validação Interna da base de conhecimentos - modelos bayesianos}

$\mathrm{Na}$ validação interna da base de conhecimentos foram utilizadas as variáveis de sensibilidade, especificidade, valor preditivo positivo e valor preditivo negativo [17]. No primeiro modelo bayesiano, observou-se que a sensibilidade do sistema em detectar que o paciente possui um evento epiléptico foi de $88,67 \%$, ou seja, 47 diagnósticos apresentando eventos epilépticos convergiram e seis diagnósticos não convergiram; a especificidade do sistema em detectar que o paciente possui um evento não epiléptico foi de 98,55\%, ou seja, 68 diagnósticos apresentando eventos não epilépticos convergiram e um não convergiu. Os resultados de valor preditivo positivo e valor preditivo negativo foram de $97,91 \%$ e $91,89 \%$, ou seja, o grau de certeza de que o evento epiléptico diagnosticado pelo sistema ser realmente positivo e do diagnóstico ser um evento não epiléptico (negativo), respectivamente. Esses resultados foram obtidos com a implementação a partir do primeiro modelo bayesiano, onde foram investigadas a variável "número total" de EEs e ENEs, a partir das 14 variáveis de entrada.

No segundo modelo bayesiano observou-se que a sensibilidade do sistema de detectar que o paciente possui um evento epiléptico foi de 79,24\%, ou seja, 42 diagnósticos apresentando eventos epilépticos convergiram e 11 não convergiram; a especificidade do sistema de detectar que o paciente possui um evento não epiléptico foi de $89,85 \%$, ou seja, 62 diagnósticos apresentando eventos não epilépticos convergiram e sete diagnósticos não convergiram. Os resultados de valor preditivo positivo e valor preditivo negativo foram de $85,71 \%$ e $84,93 \%$, ou seja, o grau de certeza de o evento epiléptico diagnosticado pelo sistema ser realmente positivo e do diagnóstico ser um evento não epiléptico (negativo), respectivamente ${ }^{4}$.

Com a implementação do terceiro modelo bayesiano, observou-se um aumento em relação aos resultados obtidos por meio do segundo modelo bayesiano: a sensibilidade do sistema em detectar que o paciente possui um evento epiléptico foi de $90 \%$, ou seja, 48 diagnósticos apresentando eventos epilépticos convergiram e cinco não convergiram; a especificidade do sistema de detectar que o paciente possui um evento não epiléptico foi de $91 \%$, ou seja, 63 diagnósticos apresentando eventos não epilépticos convergiram e seis diagnósticos não convergiram. Os resultados de valor preditivo positivo e valor preditivo negativo foram de $88 \%$ e $92 \%$, ou seja, o grau de certeza de o evento epiléptico diagnosticado pelo sistema ser realmente positivo e do diagnóstico ser um evento não epiléptico (negativo), respectivamente 5 .

Na sequência, faz-se uma comparação com os resultados obtidos através dos modelos conexionistas a partir da validação interna da base de conhecimentos. Em todas as etapas de aprendizado investigadas, os modelos conexionistas foram treinados com 1.000 épocas.

Em relação às medidas de sensibilidade, especificidade, valor preditivo positivo e valor preditivo negativo, o comportamento do aprendizado é mostrado na Tabela 1.

\footnotetext{
${ }^{4}$ Neste modelo foram investigadas as variáveis "às vezes, frequentemente e sempre", as quais foram obtidas por meio da análise realizada nos dados de entrada da rede conexionista.

${ }^{5}$ Este modelo bayesiano foi obtido segundo especificações fornecidas pelo especialista de domínio
} 
Tabela 1. Validação Interna da Base de Conhecimentos - Modelos Investigados

\begin{tabular}{|c|c|c|c|c|c|}
\hline Modelos & Regras / Operações & Sensibilidade (\%) & Especificidade (\%) & $\mathrm{VPP}(\%)$ & VPN (\%) \\
\hline RNA 1 [24] & Regra Delta Generalizada & & & 100 & 92,00 \\
\hline RNA 2 [24] & Regra de Hebb & & & 100 & 90,78 \\
\hline RNA 3 [24] & Rega de Oja & & & 95,91 & 91,78 \\
\hline $\begin{array}{l}\text { HIROTA e } \\
\text { PEDRYCZ [25] }\end{array}$ & Soma/Produto Einstein & 83,72 & & 78,26 & 90,06 \\
\hline $\begin{array}{l}\text { KWAN e } \\
\text { CAI [25] }\end{array}$ & Soma/Produto Einstein & 83,01 & & 93,61 & 88,00 \\
\hline NEFCLASS [28] & Max/Min & 84,90 & 82,60 & 78,94 & 87,69 \\
\hline Nesta pesquisa & - & - & - & - & - \\
\hline BAYESIANO 1 & $N^{\circ}$ Total & & & 97,91 & 91,89 \\
\hline BAYESIANO 2 & As vezes/Freq./Sempre & 79,24 & & 85,71 & 84,93 \\
\hline BAYESIANO 3 & Às vezes/Freq./Sempre & & & 88,00 & 92,00 \\
\hline
\end{tabular}

Observa-se a pela Tabela 1 que o melhor resultado de sensibilidade foi obtido a partir do terceiro modelo bayesiano, com $90 \%$ de acertos, seguido do primeiro e terceiro modelo conexionista e do primeiro modelo bayesiano, os três modelos com 88,67\%, juntamente com o segundo modelo conexionista o qual apresentou 87,79\% de acertos. Esses modelos apresentaram graus de certeza de 88\%, 100\%; 95,91\%; 97,91\% e 100\%, respectivamente. Em relação aos resultados de especificidade, os melhores resultados foram obtidos utilizando os modelos conexionistas um e dois (ambos com 100\%) com graus de certeza de 92\% e 90,78\%; em segundo lugar, o modelo conexionista três $(97,10 \%)$, com grau de certeza de $91,78 \%$; em seguida, o neurônio difuso Kwan-Cai (95,65\%); primeiro modelo bayesiano, com 98,55\%, e terceiro modelo bayesiano, que apresentou $91 \%$ de acertos. Os graus de certeza foram de $88 \%$; $91,89 \%$ e $92 \%$, respectivamente.

\section{Discussões}

A modelagem matemática por meio das RBs foi uma tarefa complexa, pois envolveu uma análise nas 14 variáveis de entrada da rede, as quais representaram os 122 pacientes cadastrados. A análise foi dividida pela implementação de três modelos bayesianos, utilizando conceitos da lógica booleana e da lógica difusa.

Foi necessário realizar a modelagem matemática dos modelos bayesianos dois e três porque estes modelos foram comparados com os modelos neuro-difusos baseados em Hirota-Pedrycz e Kwan-Cai e através da arquitetura NEFCLASS, os quais possuem entradas difusas (às_vezes, frequentemente e sempre). Os resultados da modelagem matemática bayesiana, relacionados com a capacidade de detectar casos negativos, foram menores aos implementados com os modelos conexionistas. É possível que esses resultados estejam relacionados com a capacidade de generalização das redes conexionistas, ou seja, caso as RBs fossem treinadas diversas vezes, como ocorre no aprendizado de RNAs, os resultados obtidos poderiam ter um percentual maior de acertos.

Na modelagem matemática bayesiana três foram utilizados os conceitos de: (1) dado que um paciente apresentou (por exemplo) "quadros clássicos de conversão", qual a probabilidade de que este sintoma contribua para que o paciente tenha um EE ou um ENE, ou seja, este percentual de certeza foi obtido segundo especificação do especialista de domínio, diferente das modelagens matemáticas bayesianas um e dois, as quais 
foram obtidas por meio de cálculos realizados na base de conhecimentos. Este fato poderá ter contribuído para que o modelo bayesiano três tenha obtido melhores resultados em relação aos modelos bayesianos um e dois.

Os resultados com os modelos conexionistas um, dois e três, juntamente com os neurônios difusos propostos por Hirota-Pedrycz e Kwan-Cai e a arquitetura NEFCLASS, foram obtidos a partir da etapa de generalização das RNAs.

Os percentuais de acertos relacionados aos modelos bayesianos foram confrontados com alguns resultados de pesquisas encontrados na literatura envolvendo RNAs, RBs e sistemas neuro-difusos, os quais são mostrados na Tabela 2.

Tabela 2. Percentuais de acertos

\begin{tabular}{lc}
\hline \multicolumn{1}{c}{ Modelos de Aprendizado de Máquina } & $\begin{array}{c}\text { Acertos } \\
\text { (\%) }\end{array}$ \\
\hline MLP - Diagnóstico de Tuberculose [5] & 80,00 \\
\hline SND - Câncer de Mama [30] & 98,28 \\
\hline SND - Diabetes em Artérias Oftálmicas [31] & 85,00 \\
\hline SND - Epilepsia [32] & 83,33 \\
\hline MLP - Tireóide [33] & 89,90 \\
\hline SND-Hirota-Pedrycz - Kwan-Cai [25] & 90,16 \\
\hline SND - NEFCLASS - Operações min/max [29] & 83,60 \\
\hline MLP 1 modificado - Delta Generalizada [24] & 95,08 \\
\hline MLP 2 modificado - Hebb [24] & 94,26 \\
\hline MLP 3 modificado - Oja [24] & 93,44 \\
\hline Rede Bayesiana - CPSG [34] & 88,00 \\
\hline Bayesiano $x$ Conexionista - Energia Elétrica [12] & 91,26 \\
\hline Rede Bayesiana - Triagem Odontológica com pacientes especiais & $913], 00$ \\
\hline Nesta Pesquisa: & 93,61 \\
\hline Modelo Bayesiano 1 & 90,05 \\
\hline Modelo Bayesiano 2 & \\
\hline Modelo Bayesiano 3 & 954 \\
\hline
\end{tabular}

Com base na Tabela 2 observa-se que os percentuais de acertos obtidos com os modelos matemáticos bayesianos um, dois e três são similares aos resultados relacionados com os modelos conexionistas e bayesianos. Dessa forma, pode-se concluir que ambos os modelos, bayesianos e conexionistas, são bastante similares, mostrando que os resultados são coerentes, mesmo utilizando heurísticas distintas.

\section{Conclusões}

Nesta pesquisa mostraram-se algumas características envolvidas na aprendizagem de máquina, entre elas a modelagem matemática bayesiana $x$ conexionista. 
A pesquisa validou diferentes regras de aprendizado, como a delta generalizada, Hebb, Oja, juntamente com dois tipos de neurônios difusos, Hirota-Pedrycz e Kwan-Cai. Os resultados obtidos demonstraram a capacidade dos modelos de trabalharem com diferentes abordagens, fornecendo resultados diferenciados na classificação de padrões. O desempenho dos modelos foi validado por meio de uma base de conhecimentos envolvendo os eventos epilépticos e os eventos não epilépticos.

Foram implementados três modelos bayesianos. Nestes modelos, verificou-se que o aprendizado em RBs está relacionado com os parâmetros da rede, ou seja, a relação das probabilidades condicionais entre as suas respectivas variáveis de entrada e de saída, ao passo que o aprendizado das RNAs está relacionado com os algoritmos de aprendizado, os quais se distinguem entre si por meio das regras de aprendizado que utilizam, envolvendo pesos de conexões em função de estímulos externos (entradas da rede). Tanto os modelos bayesianos como os conexionistas permitem a representação do conhecimento a partir de um conjunto de exemplos, razão por que ambos podem ser utilizados e validados.

O treinamento dos modelos bayesianos ocorreu pela inserção das probabilidades condicionais entre os nós, os quais possuem dependência direta, ao passo que o treinamento dos modelos conexionistas ocorreu pelo ajuste dos pesos das conexões até obter um erro aceitável. As variáveis de entrada de ambos os modelos foram as mesmas.

Considerando os resultados de sensibilidade dos modelos bayesianos um e três, observa-se que, estatisticamente, são similares ao aprendizado utilizando as regras Delta Generalizada, Hebb e Oja. Os resultados de especificidade mostram que alguns modelos obtiveram resultados semelhantes entre eles - regra Delta Generalizada, Hebb, Oja, neurônios difusos Hirota-Pedrycz e Kwan-Cai - juntamente com os modelos bayesianos um e três.

Constatou-se que os modelos bayesianos um e três, juntamente com os modelos conexionistas um, dois e três, tiveram resultados semelhantes para diagnosticar eventos epilépticos. Em relação ao diagnóstico de eventos não epilépticos, verificou-se que a maioria dos modelos, tanto bayesianos como conexionistas apresentou resultados compatíveis.

Os modelos investigados fornecem informações importantes para o desenvolvimento de novas pesquisas tanto na área bayesiana como na área conexionista. Os índices de acertos de casos positivos (EEs) e casos negativos (ENEs) segundo a modelagem bayesiana podem estar relacionados com a facilidade de implementação das probabilidades condicionais. Uma das vantagens das RBs é a possibilidade de mostrar com maior clareza essas probabilidades, facilitando o processo de manutenção.

Portanto, conclui-se:

(1) que os modelos bayesianos um e três sejam utilizados em conjunto com os modelos conexionistas que utilizam as regras de aprendizado Delta Generalizada, Hebb e Oja no diagnóstico complementar de eventos epilépticos;

(2) que os modelos bayesianos um e três sejam utilizados em conjunto com os modelos conexionistas, que utilizam as regras de aprendizado Delta Generalizada, Hebb, Oja juntamente com os neurônios difusos Hirota-Pedrycz e Kwan-Cai, no diagnóstico complementar de eventos não epilépticos.

Concluindo, com base nesta investigação, constata-se, que, ao desenvolver um sistema de apoio à tomada de decisão envolvendo a área da inteligência artificial, devem ser aplicados diferentes modelos de aprendizagem de máquina na tentativa de simular com maior precisão o raciocínio humano. Como trabalhos futuros, sugere-se: (1) fazer a validação concorrente a partir dos modelos investigados em áreas distintas do conhecimento; (2) pesquisar outras topologias de modelos conexionistas que permitam identificar semelhanças com a modelagem bayesiana.

\section{Referências}

[1] LIMA, C.A.M.; COELHO, A.L.V.; CHAGAS, S. Automatic EEG signal classication for epilepsy diagnosis with Relevance Vector Machines. Expert Systems with Applications, v. 36, p. 10054-10059, 2009.

[2] GUERREIRO, C. A. M. et al. Epilepsia. São Paulo: Lemos Editorial, 2000. 
[3] NIGAM, V. P.; GRAUPE, D. A neural network based detection of epilepsy. Neurological Research, v. 26, p 55-60, 2004.

[4] VELASCO, M. M. B. R.; PACHECO, M. A. C. In: NÚCLEO DE PESQUISA EM INTELIGÊNCIA COMPUTACIONAL APLICADA. PUC-RIO. Disponível em: <http://www.ica.ele.puc-rio.br/home/index.rails>. Acesso em: 9 maio 2009.

[5] ANTUNES, P. H. S.; SEIXAS, J. M. de; MAIDANTCHIK, C. Redes neurais aplicadas ao diagnóstico da tuberculose pulmonar paucibacilar. In: CONGRESSO BRASILEIRO DE REDES NEURAIS, 8, Rio de Janeiro. Anais... 2007.

[6] SANTOS, A. M.; SILVA, A. C.; MARTINS, L. O. A neural-bayesian approach for predicting smear negative pulmonary tuberculosis. In: CONGRESSO BRASILEIRO DE REDES NEURAIS, 8, Florianópolis, Santa Catarina. Anais... 2007.

[7] TEZEL, G.; OSBAY, Y. A new approach for epileptic seizure detection using adaptative neural network. Konya, Turkey, 2009.

[8] SANTOS, A. M. et al. Usando redes neurais artificiais e regressão logística na predição da Hepatite A. Rio de Janeiro: Universidade Federal do Rio de Janeiro, 2005.

[9] OLIVEIRA, F. R. de. Rede neural difusa com t-normas diferenciáveis e interativas. 2006. Dissertação (Mestrado em Ciência da Computação) - Universidade Federal do Mato Grosso do Sul, Campo Grande.

[10] CARVALHO, L. M. F. de et al. A neuro-fuzzy system to support in the diagnostic of epilepsy events and non-epileptic events using different fuzzy arithmetical operations. Arq. Neuropsiquiatria, v. 66, p.179-183, 2008.

[11] NASSAR, S. M.; STEIN, C. Sistema Especialista com Base de Conhecimentos. In: WORKSHOP DE INFORMÁTICA APLICADA À SAÚDE, 2, Itajaí, Brasil. Anais... 2002. p. 1-5.

[12] TRONCHONI, A.B. Identificação de causas de desligamentos não programados em redes de distribuição. 2008. Dissertação (Mestrado em Engenharia Elétrica) - Pontifícia Universidade Católica do Rio Grande do Sul, Porto Alegre.

[13] BELTRÃO, C. J.; SCHMITT, E. J.; DIAS, J. da S. Utilização de Rede Bayesiana para auxílio de tomada de decisão na triagem de atendimento odontológico. In: WORKSHOP DE INFORMÁTICA APLICADA À SAÚDE, 2. Itajaí, Brasil. Anais... 2002.

[14] LÓPEZ, P. J.; GARCIA, G., J.; FUENTE, S. et al. Bayesian Nets as modelling tools in psychology. An. Psiscol., v. 23, n. 2, p. 307-316, 2007.

[15] DUTRA, I.; MARQUES, R. L. Redes Bayesianas: o que são, para que servem, algoritmos e exemplos de aplicações. Rio de Janeiro: Universidade Federal do Rio de Janeiro, 1999.

[16] RICH, E.; KNIGHT, K. Inteligência artificial. 2. ed. São Paulo: Makron Books, 1994.

[17] NASSAR, S. M. Estatística e informática: um processo interativo entre as duas ciências. Florianópolis, Brasil: Universidade Federal de Santa Catarina, 1998.

[18] PEARL, J. Probabilistic Reasoning in Intelligent Systems: networks of plausible inference. California: Morgan Kaufmann Publishers Inc., 1988. 552 p.

[19] NETICA. Shell para sistema especialista probabilistico. Disponível em: <http://www.norsys.com>. Acesso em: 14 jul. 2009. 
[20] BRAGA, A. P.; CARVAlho, A. P. L. F. de; LUDERMIR, T. B. Redes neurais artificiais: teoria e aplicações. 2. ed. Rio de Janeiro: LTC, 2007.

[21] PAULA, M. B. de. Indução automática de árvores de decisão. Florianópolis, 2002.

[22] HAYKIN, S. Redes neurais: princípios e prática. 2. ed. Porto Alegre: Bookman, 2001.

[23] WIDROW, B.; HOFF, M. E. Daptative Switching circuits. Instiute of Radio Engineers. In: WESCON CONVENTION RECORD, Part 4, p. 96-104, 1960.

[24] SCHWALBERT, M. A. Metodologias de aprendizagem de máquina utilizando Redes Neurais Artificiais. 2007. Monografia (Bacharel em Ciência da Computação) - Universidade de Passo Fundo, Passo Fundo.

[25] MONTEIRO, L. L. Análise e validação de um sistema neuro-difuso na área médica. 2005. Monografia (Bacharel em Ciência da Computação) - Universidade de Passo Fundo, Passo Fundo.

[26] HIROTA, K.; PEDRYCZ, W. Knowledge-based networks in classification problems. Fuzzy Sets and Systems, v. 59, n. 3, p. 271-279, 1993.

[27] KWAN, H. K.; CAI, T. A Fuzzy Neural Network and its Application to Pattern Recognition. IEEE Transactions on Fuzzy Systems, v. 3, p. 185-193, 1994.

[28] CARVAlHO, L. M. F. de. Modelos de Aprendizagem para Sistemas Neuro-Difusos. 2007. Tese (Doutorado em Engenharia Elétrica) - Universidade Federal de Santa Catarina, Florianópolis.

[29] NECLASS. Shell para Sistemas Neuro-Difusos. Disponível em: <http://fuzzy.cs.unimagdeburg.de/nefclass/>. Acesso em: 14 jul. 2009.

[30] CONDE, G. A. B.; RAMOS, P. G.; VASCONCELOS, G. C. Redes neuro-difusas para classificação de padrões e extração de regras In: SIMPÓSIO BRASILEIRO DE REDES NEURAIS, 6, Rio de Janeiro. Anais... 2000.

[31] SERHATLIOGLU, S. et al. Investigation of the influence of training status on the relationship between the acute exercise and serum leptin levels in obese females. Neuroendocrinology Letters, 2004.

[32] BRASIL, L. M. Proposta de Arquitetura para Sistema Especialista Híbrido e a Correspondente Metodologia de Aquisição do Conhecimento. 1999. Tese (Doutorado em Engenharia Elétrica) Universidade Federal de Santa Catarina, Florianópolis.

[33] RAMOS, F. R. Implantação de um sistema especialista probabilístico. 2001. Monografia (Bacharel em Ciência da Computação) - Universidade de Passo Fundo, Passo Fundo. 